\title{
A wavelet analysis of quasi-periodic variability across a solar coronal hole region
}

\author{
M. S. Marsh, R. W. Walsh, and B. J. I. Bromage \\ Centre for Astrophysics, University of Central Lancashire, Preston, PR1 2HE, UK \\ Received 3 May 2002 / Accepted 18 July 2002

\begin{abstract}
High time resolution $(\approx 13 \mathrm{~s}$ cadence) Solar and Heliospheric Observatory (SOHO) Coronal Diagnostic Spectrometer (CDS) Grazing Incidence Spectrometer (GIS) time series data is analysed for evidence of quasi-periodic variability in the solar corona. The observations are obtained from a GIS study of a coronal hole boundary region taken in October 1997. A wavelet analysis is applied to EUV emission lines covering a temperature range of $\log T_{\mathrm{e}}=5.3-6.1 \mathrm{~K}$. Wavelet analysis allows the frequency and temporal information of any periods present within the data to be analysed. Statistically significant periods are found within the range 100-900 s and $1500 \mathrm{~s}$. Observations of short wavepackets with periods of the order 50-100 s with durations of $2-5$ cycles are reported. Observations of a decay in the driving period over time are also described.
\end{abstract}

Key words. Sun: corona - Sun: oscillations - Sun: transition region - Sun: UV radiation - waves

\section{Introduction}

Understanding the complex temperature structure of the solar corona is still an unresolved problem in modern astrophysics. However, it is generally accepted that the processes involved in heating the solar atmosphere above the chromosphere are magnetic in origin. A number of theoretical heating mechanisms have been proposed in the literature (Hollweg 1990; Narain \& Ulmschneider 1990; Zirker 1993), many of which involve the dissipation of MHD wave energy at coronal heights. Observational evidence of oscillations in the solar atmosphere is required to provide constraints on the theory of heating mechanisms.

The observations of oscillations in the corona have largely been interpreted as slow magneto-acoustic mode or kink mode oscillations. Aschwanden et al. (1999) report the first observation of kink mode oscillations in a coronal loop, and more recently, Nakariakov \& Ofman (2001) develop a method of determining the magnetic field strength in coronal loops by using the kink mode period and loop length. DeForest \& Gurman (1998) report on the observation of compressive wave trains in polar plumes; it is suggested that these may be caused by the presence of magneto-acoustic waves. Recent results on observations that are interpreted as slow magneto-acoustic modes are described by Ireland et al. (1999), De Moortel et al. (2000), Banerjee et al. (2001a), Hansteen et al. (2001), O'Shea et al. (2001), Robbrecht et al. (2001), commonly finding periods in the range 150-500 s. Many of these recent studies use wavelet analysis; essentially this is an extension of Fourier analysis into

Send offprint requests to: M. S. Marsh,

e-mail: mmarsh@uclan.ac.uk the time-frequency domain allowing the time dependence of the periods present to be studied.

In this paper we apply wavelet analysis to Coronal Diagnostic Spectrometer (CDS) Grazing Incidence Spectrometer (GIS) (see Harrison et al. 1995) observations of a network-internetwork area located near a coronal hole boundary region. The technique is applied to total line intensity time series formed from the eight coronal/transition region EUV emission lines listed in Table 1. The campaign designed to study the coronal hole boundary regions is described in Sect. 2. The data reduction and processing is detailed in Sect. 3 with the results presented in Sect. 4 and discussion following in Sect. 5.

\section{Observations}

The observations analysed in this paper were obtained from a CDS-GIS study performed on 15th October 1997 at 21:21 UT. This study contained fifteen positions of the GIS slit, beginning at a coronal hole boundary and crossing into the coronal hole in the West-East direction. At each position 100 exposures of $5 \mathrm{~s}$ duration were taken resulting in a cadence of approximately $6 \mathrm{~s}$ after taking into account instrumental overheads. The study uses the $4^{\prime \prime} \times 4$ " slit, stepping in the West-East direction by 4 arcsec increments for each successive position. It should be noted that the GIS grating is astigmatic i.e. images are not resolved spatially. Therefore the GIS spectra are one-dimensional.

Figure 1 shows NIS images in the Ov $629.7 \AA$ line (peak emission at a temperature of $\log T_{\mathrm{e}}=5.4 \mathrm{~K}$ ) and the Mg IX $368 \AA$ line (peak emission at $\log T_{\mathrm{e}}=6.0 \mathrm{~K}$ ) showing 

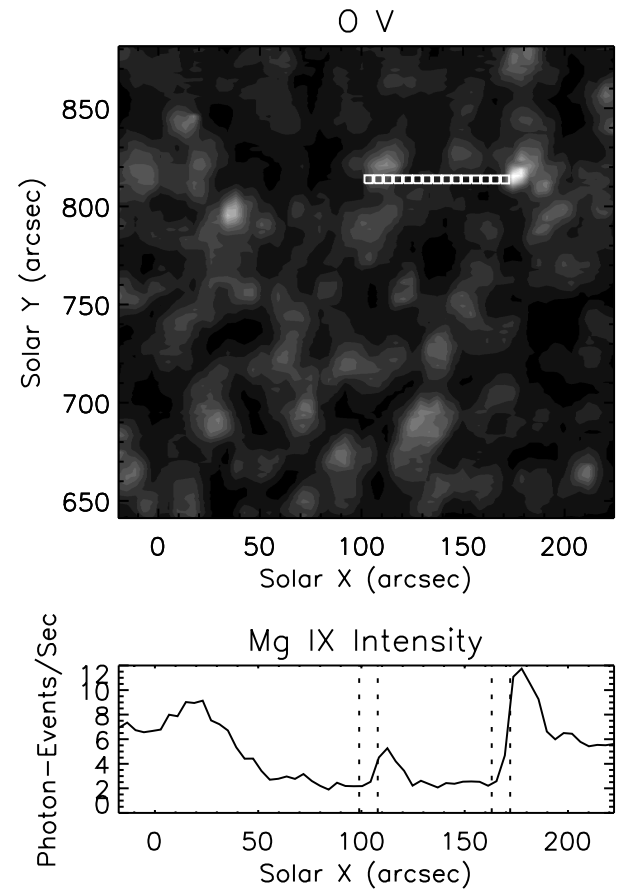

$\mathrm{Mg}$ IX

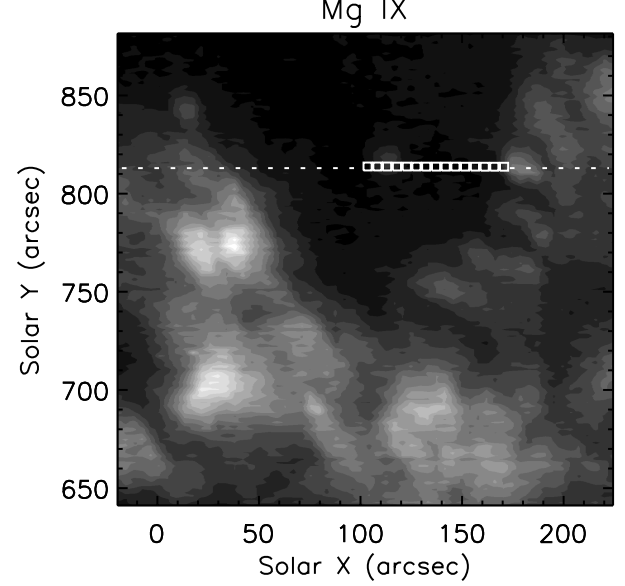

Fig. 1. Positions of the GIS observations overlying Ov $629 \AA$ and Mg IX $368 \AA$ NIS context images. The observing sequence runs from right to left. The $\mathrm{O} v$ image reveals the chromospheric network pattern as high intensity regions. The GIS observations begin and end in the network, with most of the sequence passing over the inter-network regions. The $\mathrm{Mg}$ IX image displays the location of the coronal hole. The GIS observations begin at the hole boundary and end within the centre of the hole. The middle plot shows Mg Ix intensity along dotted line in the $\mathrm{Mg}$ IX context image. The vertical dotted lines mark the location of the first and last two GIS observing positions which lie over the two network regions. The hole boundary appears at around $40^{\prime \prime}$ and $170^{\prime \prime}$ with the lower intensity coronal hole region in between.

the chromospheric network pattern and the coronal hole region respectively. Plotted over the images are the 15 positions of the $4^{\prime \prime} \times 4^{\prime \prime}$ GIS slit with each box representing the slit boundary. The NIS observations preceded the GIS observations by $\approx 40 \mathrm{~min}$. This has been taken into account with respect to solar rotation when plotting the slit positions. The $\mathrm{O} v$ image shows the positions of the observations relative to the bright and dark patches corresponding to the network and inter-network regions respectively. The Mg IX image shows the positions of the
Table 1. Spectral lines analysed with emitting ion, emitted wavelength, and log of peak emission temperature.

\begin{tabular}{lll}
\hline \hline Ion & Wavelength $\AA$ & $\log T_{\mathrm{e}}$ \\
\hline Ne IV & 469.9 & 5.31 \\
Ne vII & 465.2 & 5.88 \\
Ne vIII & 780.3 & 5.95 \\
Ne VIII & 770.4 & 5.96 \\
O VI $_{\text {Ve }}$ & 173.0 & 5.97 \\
Fe XI & 171.1 & 6.00 \\
Fe xIII & 180.4 & 6.05 \\
\hline
\end{tabular}

observations relative to the coronal hole region. The middle plot in Fig. 1 shows the Mg IX intensity across the dotted line in the Mg IX context image. This displays the lower intensity region of the coronal hole and the brighter quiet sun regions. The position of the coronal hole boundary region can be estimated from the extent of the intensity transition between the coronal hole and quiet sun regions. The vertical dotted lines in this plot show the location of the first and last two positions of the GIS observations. These are located over the bright network regions with the first two also positioned over the coronal hole boundary region. The slight misalignment between these positions and the network regions can be accounted for by the CDS pointing error $\left(\approx 5^{\prime \prime}\right)$ and the time delay between the NIS and GIS observations.

\section{Analysis}

\subsection{The GIS spectra}

\subsubsection{Instrumental effects}

GIS suffers from a number of instrumental effects which are described in more detail in the CDS software notes 54, 55, and 56 (Bentley 1999; Breeveld 2000a,b). "Ghosting" is one of the instrumental effects that occurs because the analogue electronics in the GIS detectors are affected by electronic noise. This results in an uncertainty in the position of an event on the detector. Consequently, counts occurring at one position on the detector can be wrongly recorded as occurring in a different part of the spectrum. However, the regions of the spectrum that are affected (ghosting regions) can be calculated. Standard CDS software procedures are available to display the ghosting regions (Fig. 2), and if possible "shift" the ghosted counts back to their correct position (see CDS software notes 54-56). For this analysis it was decided to avoid spectral lines located in ghosting regions completely, thus eliminating any effect of ghosting, or its correction, on the intensity of the lines. However, this restricts the number of lines available for analysis; the lines analysed should also be isolated, unblended and have maximum signal to noise. Considering these criteria the lines were identified and selected using Del Zanna (1999). The lines selected for analysis are listed in Table 1.

Another instrumental effect, namely "Fixed Patterning" is due to inadequate resolution in the calculation of the events 


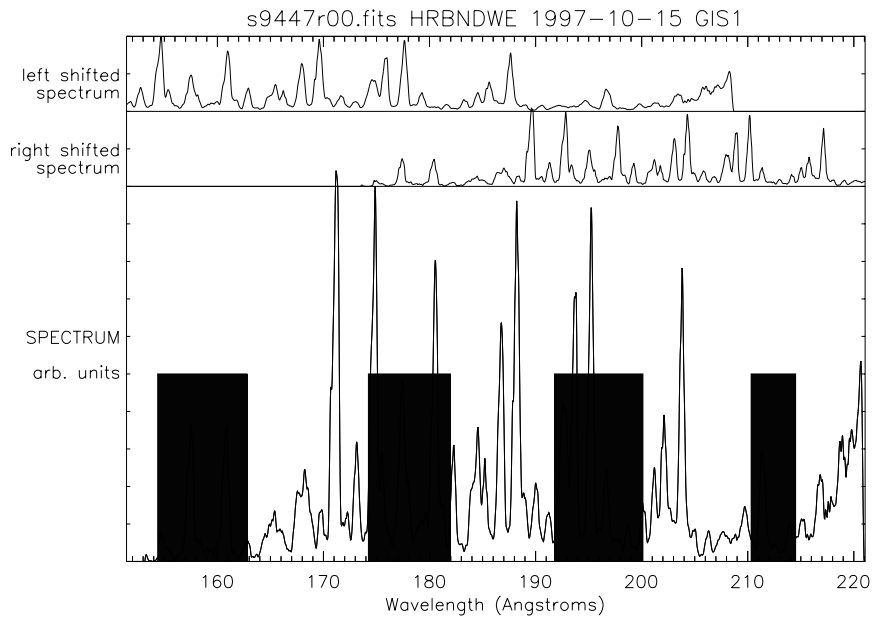

Fig. 2. Single detector spectrum with bars indicating ghosting regions.

position on the detector. This means that adjacent pixels can share varying amounts of counts i.e. some pixels can show enhanced counts while others show a reduced number of counts. Fixed patterning can be reduced by smoothing the spectral data.

The original data were taken at a cadence of $\approx 6 \mathrm{~s}$. However the data were summed over two exposures to increase the signal to noise ratio giving a cadence of $\approx 13 \mathrm{~s}$.

\subsubsection{Calibration}

Data is normally calibrated using the standard CDS software routine GIS_CALIB. This applies corrections for the various dead times and non-linearities across the GIS detector. The current observations are of a low intensity quiet-sun/coronal hole region; thus dead time corrections should have little effect. Also the analysis is concerned with variations in intensity so the absolute intensity need not be calculated. Since small variations in intensity are being investigated and the calibration applies unnecessary modifications to the data (which may introduce errors affecting the detected periodicities) no intensity calibration is applied in this analysis. Any cosmic ray strikes in the data will be visible in the time series formed as a saturation in intensity. Pre-launch wavelength calibrations are applied automatically when the data is read.

\subsection{Time series}

\subsubsection{Simple pixel summing method}

The time series in this study were formed using the simple method of summing the number of counts across the spectral pixels covered by each line. The exposures from the first position in the study showed no significant Doppler motion of the lines. It is also found that the wavelet analysis is quite insensitive to summing within the ranges used close to the line. The time series were formed by summing across each emission line with a pixel range that encompasses the entire line to allow for any slight Doppler variation that might be present. A method of determining the lines intensity by curve-fitting was excluded as the data would have to be smoothed to reduce the fixed

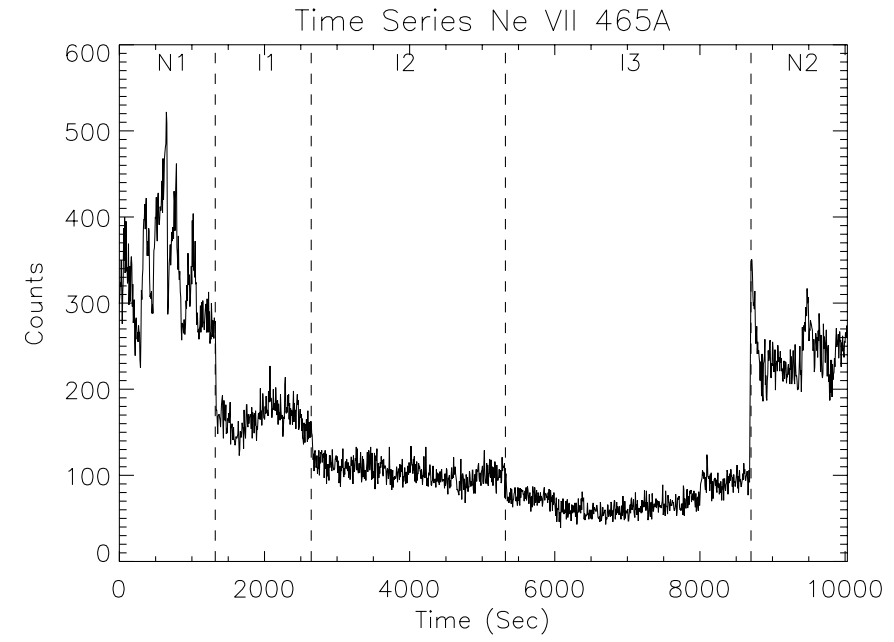

Fig. 3. Ne vII $465 \AA$ time series for whole study. The dotted lines indicate where the time series is broken down into blocks with two network (N1, N2) and three inter-network (I1, I2, I3) regions. Note that progression in time corresponds to slit movement from West to East across the sun. Each of the 15 slit positions is observed for approximately $660 \mathrm{~s}$.

patterning. This would also smooth more noise into the lines estimated intensity. The process itself would also introduce fitting errors and would also be increasingly unreliable for low intensity lines. The simplest and most accurate method of determining the lines total intensity appears to be one of summing the counts across a pixel range, on condition that the line does not show significant Doppler motion (see Sect. 3.2.2). The background level is $\approx 0.1$ photon-events/pixel/sec with slight variation across the different detectors. In this analysis the background is assumed to be negligible relative to the intensity of the lines and is assumed to be zero.

Figure 1 indicates that the observing sequence begins in a bright network region, passes through an internetwork region and ends in a less intense network region. The first slit position is located at the western boundary of a coronal hole with the rest of the positions located within the hole itself. These changes in structure imply that a concatenated time series formed from the whole study would show variations in intensity representing different structures over which the slit is positioned. Figure 3 shows the concatenated time series for the whole study in the Ne VII $465 \AA$ line. Clearly visible are the bright network regions at the beginning and end of the time series, along with the low intensity inter-network region in between.

As described in Sect. 3.3.1 the wavelet analysis is performed assuming Poisson distributed noise. To make this assumption the time series must be considered as stationary i.e. the time series must have a constant mean and variation. From Fig. 3 clearly this is not the case for the entire time series. To perform the analysis the time series must be broken down into blocks that show similar structure. It is then assumed that these time series blocks are stationary. Considering Fig. 3 it can be seen that the network regions at the beginning and end of the time series can be split into two separate time series without discontinuities. The internetwork region can also 
be split into three separate conterminous time series with each analysed separately. As each spectral line has a different emission temperature the time series for each line is broken down into different size blocks representing changing structure with emission temperature. Also note that the maximum measurable period is limited by the length of the time series being analysed.

\subsubsection{Curve fitting of $\mathrm{Ne} \mathrm{vII} \mathrm{and} \mathrm{Ca}$ IX}

It was found that the Ne VII $465.2 \AA$ line is situated close to the Ca Ix $466.2 \AA$ line. Although neither of the lines appear to show any significant Doppler motion, a curve fitting method was applied to the data to determine the intensity of the Ne vII line. This was applied using the standard CDS software procedure CFIT_BLOCK. Multiple Gaussians can be fitted to the data allowing the intensity of the Ne vir line to be isolated from any effect the Ca Ix line may have. A 10pt smooth was first applied to the data to reduce the fixed patterning - this was to reduce any fitting errors produced by the automated routine that CFIT_BLOCK employs. The time series obtained by this Gaussian fitting method and its associated wavelet power spectrum were compared to those produced by the simple summing method and found to be almost identical.

As mentioned previously, before Gaussian fitting is applied to GIS data it must first be smoothed to reduce the fixed patterning. This allows the possibility that some of the counts from Ca IX may be smoothed into the Ne vII line and vice versa. To eliminate this effect as a possibility, another test was performed on extracting the time series. This method used the fact that under a 10pt smooth any counts merged into the Ne vir line will cause an asymmetry in the right hand side of the lines profile. To determine the intensity of the Ne VII line, the centre of the line is found by the fact that points either side of it should show the minimum amount of asymmetry. The counts in the left hand side of the line can then be determined and doubled to reproduce the total counts within the line. Again the time series and wavelet power spectrum were almost identical to those produced by the simple summing method, giving agreement within the level of noise.

This demonstrates that the Ca Ix line has a negligible blending effect on the Ne vII line and that the simple summing method is an acceptable method of extracting the time series.

\subsection{Wavelet analysis}

Unlike the Fourier transform, which returns frequency information on the time series as a whole, the wavelet transform returns wavelet scale information as a function of time. Wavelet scale is proportional to the Fourier period and is easily calculated. Consequently, wavelet analysis allows the investigation of the time dependence of periods within the data. This makes the technique useful for investigating periods that are localised in time.

A detailed description of the wavelet transform and wavelet analysis is given in Torrence \& Compo (1998); a summary of the method is presented here. Assuming a time series $x_{n}$ of $N$ observations and constant sample spacing $\delta t$, the continuous wavelet transform is defined as the convolution of $x_{n}$ with a scaled and translated wavelet (or mother) function $\psi(\eta)$, where $\eta$ is a non dimensional time parameter. Acceptable wavelet functions are localised in time and frequency space with zero mean, thus we assume $\psi$ is normalised, i.e. $\int_{-\infty}^{+\infty} \psi \psi^{*} \mathrm{~d} \eta=1$. As in other works (Ireland et al. 1999; De Moortel et al. 2000) we use the Morlet wavelet as our wavelet function. The Morlet consists of a plane wave modulated by a Gaussian;

$\psi(\eta)=\pi^{-1 / 4} \exp (i 6 \eta) \exp \left(-\frac{\eta^{2}}{2}\right)$.

The continuous wavelet transform is defined as

$W_{n}(s)=\sum_{n^{\prime}=0}^{N-1} x_{n^{\prime}} \sqrt{\frac{\delta t}{s}} \psi^{*}\left[\frac{\left(n^{\prime}-n\right) \delta t}{s}\right]$,

where $\eta=\left(n^{\prime}-n\right) \delta t / s, s$ is the wavelet scale and $n$ allows the wavelet function to be translated in time. Varying the wavelet scale $s$ and translating along the time index $n$ allows a twodimensional power spectrum to be formed. This describes how the periods present vary with scale and as a function of time. The wavelet power spectrum is then defined as $\left|W_{n}(s)\right|^{2}$.

The time series and wavelet function are finite. This means that the wavelet transform suffers from edge effects at the ends of the time series by an amount proportional to the scale. A cone of influence (COI) indicates where these edge effects become significant; this is defined by the region in which the wavelet power drops by a factor of $e^{-2}$. Areas of the wavelet power spectrum outside the region bounded by the cone of influence and the time axis suffer from these edge effects and should not be included in any analysis.

\subsubsection{Significance levels}

To place a confidence or significance level on any periods present in the wavelet power spectrum, a background noise spectrum must be assumed. Thus any power present in the analysis is calculated by comparing the data power spectrum to the background noise spectrum (Ireland et al. 1999; De Moortel et al. 2000) Contours of significance are formed, inside which the wavelet power can be considered as being real within a given confidence level. We assume Poisson distributed noise from the photon counting statistics. Then assuming a stationary time series $x_{n}$ of $N$ observations, the Poisson noise is given by $\sigma_{\mathrm{P}}^{2}=\bar{x}$, where $\bar{x}=\frac{1}{N} \sum_{i=1}^{N} x_{i}$. For this analysis we chose a significance level of $99.0 \%$, implying that there is a $1.0 \%$ probability that any significant power is caused by chance.

\subsubsection{Evenly spaced time series}

As mentioned briefly in Sect. 3.3 wavelet analysis requires evenly sampled data. Although the exposure time for this study is $5 \mathrm{~s}$, after instrument overheads are taken into account this is increased. Examination of the studies timing data shows that the average exposure spacing is $6.6 \mathrm{~s}$ with a standard deviation of $0.23 \mathrm{~s}$. This leads to a $3 \%$ fractional error in the cadence 


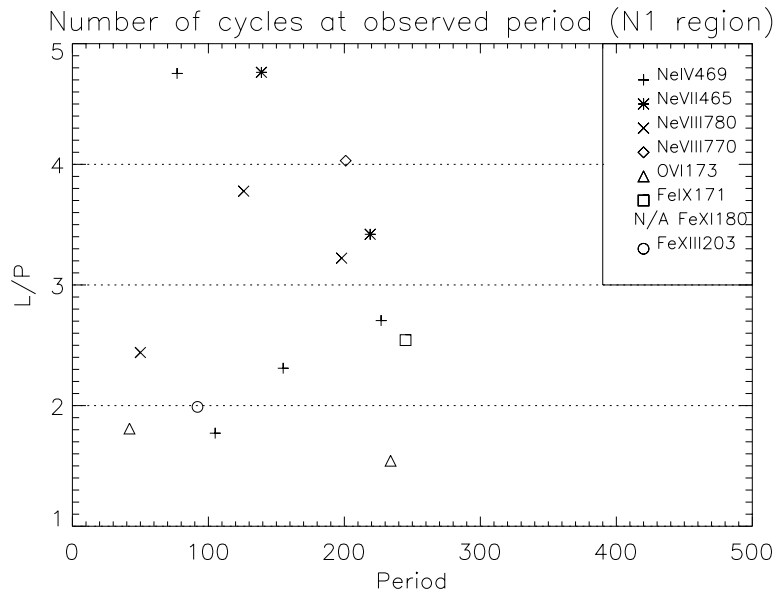

Fig. 4. Number of cycles observed $(L / P)$ for each period in the first network region N1. $L$ is the extent of the period in time, $P$ is the period.

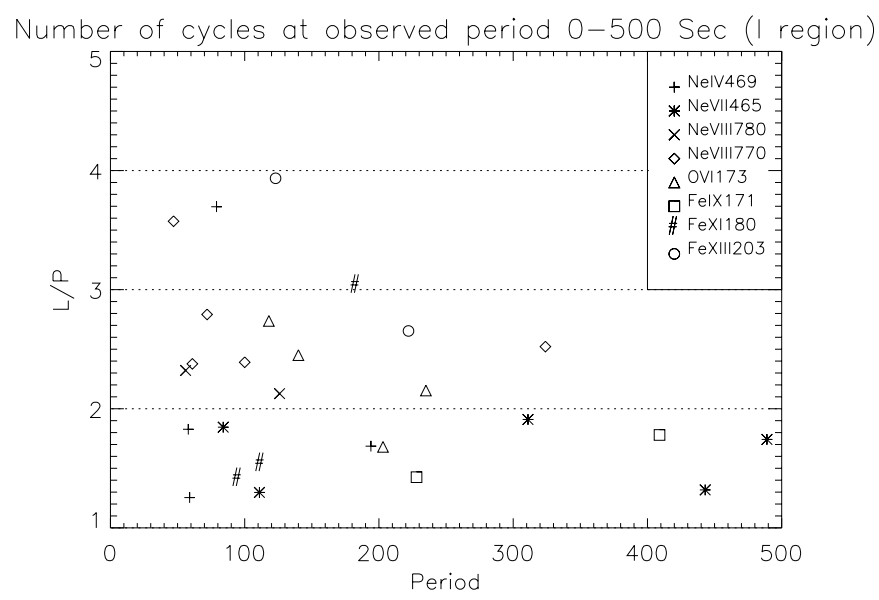

Fig. 5. $(L / P)$ in the inter-network region I. Period range 0-500 s.

of the study and any significant periods detected. Any irregular spacing in time between slit positions is linearly interpolated by rounding down to an integer spacing of this mean cadence. Typically, adjacent slit positions are interpolated using 1 or 2 data points. Any localised periods occurring at a boundary between positions are indicated in the results to be aware of any effect which non-integer spacing may cause.

\section{Results}

The results presented here were obtained from observations taken on the 15th of October 1997. Table 2 lists the results from the wavelet analysis which were read directly from the wavelet power spectra. Listed in the table are the occurrence times from the beginning of the study and the value of any periods present in the data. The stated values indicate the period and time of maximum wavelet power (Max). Wavelet analysis does not return an exact value of period or time; instead contours of significance are formed around any power lying within a given significance level as shown in Figs. 8 and 9. The ranges given in the table indicate the extent of these significance levels

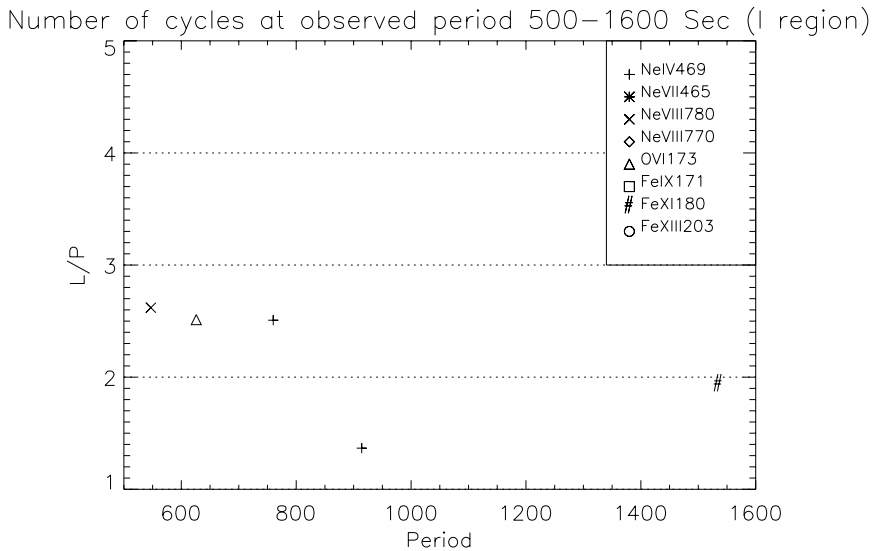

Fig. 6. $(L / P)$ in the inter-network region I. Period range 500-1600 s.

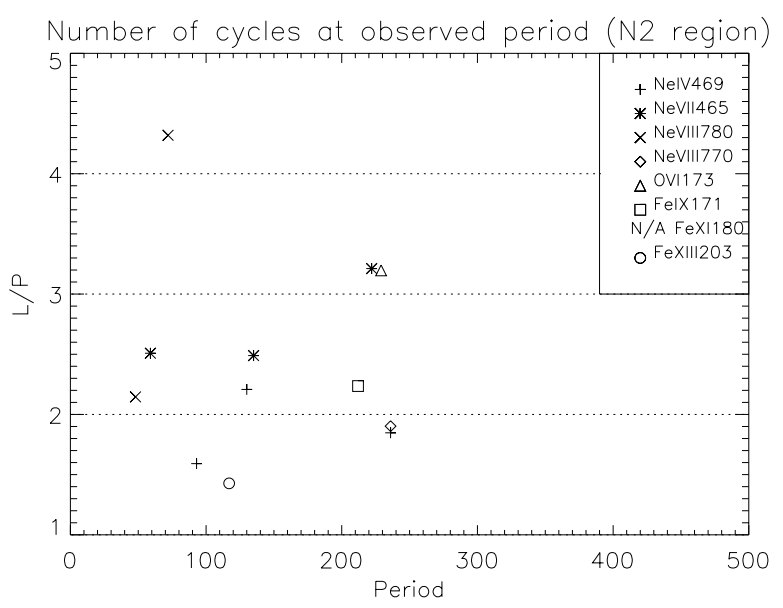

Fig. 7. $(L / P)$ in the second network region N2.

along the period and time axes. In cases where the contour levels for a particular period fall outside the COI, then the ranges limited by the COI are given and indicated by (c). The periods present in the spectra were divided into two classes: bands (b) and localised (o) bursts. These are either bands of power that extend across the COI or localised bursts of power that persist for only a few cycles. In the cases where no (Max) is given only the lower values of the period range can be valid given that the time range within the COI must be long enough to allow one complete cycle. Results marked with a $\dagger$ occur at points in the time series where the interval between positions has been interpolated. The physical region in the study where the period is observed is also listed. Positions $00-01$ cover the first network region (N1), positions 13-14 are located over the second network region (N2) and positions $02-12$ overlie the inter-network region.

Table 3 lists some of the most recent work on periodicities in the transition region and corona along with the observed region and the dominant periods found. We present periods in Table 2 that are consistent with these across the different physical regions.

The temporal information that wavelet analysis returns allows other properties of the detected periods to be determined. 
Table 2. Wavelet results for each spectral line including period and time of occurrence from the beginning of the study. Ranges indicate limits of contours formed by $99 \%$ confidence level. Values limited by COI are indicated by (c). (Max) indicates period and time values of maximum wavelet power. Periods are classed as either bands (b) or localised bursts (o). $\dagger$ indicates periods that occur on a boundary between slit positions in the time series. The physical location in which the period occurs is listed as either the first/second network region (N1/N2) or the inter-network region (I). Periods and times are given in seconds.

\begin{tabular}{|c|c|c|c|c|}
\hline Ion Wavelength $\AA$ & Period Range (Max) & Time Range (Max) & Class & Location \\
\hline $\mathrm{Ne}$ IV 469 & $48-99(77)$ & $266-632(451)$ & o & N1 \\
\hline- & $94-117(105)$ & $988-1174$ (1091) & o & $\mathrm{N} 1$ \\
\hline - & $96-233(155)$ & $540-898(703)$ & $\mathrm{b}$ & $\mathrm{N} 1$ \\
\hline - & $180-342(227)$ & 314-928 (317) & $\mathrm{b}$ & $\mathrm{N} 1$ \\
\hline - & $171-225$ (194) & $4038-4365$ (4187) & o & I \\
\hline - & $281-1445 c(760)$ & $2370 c-4276 c(3233)$ & $\mathrm{b}$ & I \\
\hline - & $54-64(59)$ & 5930-6004 (5967) & o & I \\
\hline - & $50-66(58)$ & $8490-8596 c(8558)$ & $\mathrm{o}$ & I \\
\hline - & $66-93(79)$ & $8185-8477$ (8328) & $\mathrm{o}$ & I \\
\hline - & 753-1081 (914) & $6377 c-7626 c$ & $\mathrm{~b}$ & I \\
\hline - & $76-113(93)$ & 9324-9472 (9389) & $0 \dagger$ & $\mathrm{N} 2$ \\
\hline - & $113-142(130)$ & $9545-9856 c$ (9828) & $\mathrm{b}$ & $\mathrm{N} 2$ \\
\hline - & $203-262(236)$ & $8988 c-9424$ & $\mathrm{~b}$ & $\mathrm{~N} 2$ \\
\hline- & $336-482 c$ & $9177 \mathrm{c}-9552 \mathrm{c}$ & $\mathrm{b}$ & $\mathrm{N} 2$ \\
\hline Ne VII 465 & $40-90(66)$ & $576-749(656)$ & $0 \dagger$ & N1 \\
\hline- & $101-169$ (139) & $219-881(685)$ & o & $\mathrm{N} 1$ \\
\hline - & $184-273$ (219) & $283 c-1032(390)$ & $\mathrm{b}$ & N1 \\
\hline - & $243-367$ (311) & $1671 c-2265 c(1793)$ & $\mathrm{b}$ & I \\
\hline - & $96-129(111)$ & $4602-4746(4667)$ & $0 \dagger$ & I \\
\hline - & $435-551$ (489) & $3874-4726 c(4345)$ & $\mathrm{b}$ & I \\
\hline - & $40-106(84)$ & $7993-8148$ (8067) & $0 \dagger$ & I \\
\hline - & $148-687$ & $7440-8341 \mathrm{c}(8018)$ & $\mathrm{o}$ & I \\
\hline - & $356-529(443)$ & $5849 c-6433(6079)$ & o & I \\
\hline - & $39-84(59)$ & $8825-8973$ (8899) & o & $\mathrm{N} 2$ \\
\hline - & $85-143$ (135) & 9496-9832 (9794) & $\mathrm{b}$ & $\mathrm{N} 2$ \\
\hline- & $178-274(222)$ & $9002-9715$ (9491) & $\mathrm{b}$ & $\mathrm{N} 2$ \\
\hline Ne VIII 780 & $42-58(50)$ & $302-424(358)$ & o & $\mathrm{N} 1$ \\
\hline- & $116-138$ (126) & $324-800(517)$ & $\mathrm{b}$ & $\mathrm{N} 1$ \\
\hline - & $180-255$ (198) & $398-1036(734)$ & $\mathrm{b}$ & N1 \\
\hline - & $44-73(56)$ & $2174-2304(2230)$ & o & I \\
\hline - & $101-156(126)$ & $2860-3128(2983)$ & o & I \\
\hline - & $405-671(547)$ & $4102-5535(4920)$ & o & I \\
\hline - & $1458-1894$ & $4707 \mathrm{c}-6577 \mathrm{c}(5804)$ & $\mathrm{b}$ & I \\
\hline - & $39-57(48)$ & $9000-9103$ (9057) & o & $\mathrm{N} 2$ \\
\hline - & $61-85(72)$ & 9599-9910c(9690) & o & $\mathrm{N} 2$ \\
\hline
\end{tabular}

The number of cycles of a given period can be obtained from the wavelet power spectra. Here the given period is that having maximum wavelet power within the contour level, and the extent of the period in time is given by the range in time of the contour level e.g. a period of $200 \mathrm{~s}$ persisting for $800 \mathrm{~s}$ has 4 detected cycles. Figures 4, 5, 6 and 7, show the number of cycles observed for each detected burst of periodicity in the first network region (Fig. 4), inter-network region (with same period range as network regions Fig. 5), inter-network region with longer period range (Fig. 6) and second network region (Fig. 7). It should be noted that the number of cycles in the longer periods may be limited by the COI depending on the length of the time series, particularly in the network regions.
With this in mind, to produce a stationary signal the time series for Fe XI had to be formed from single positions in the network regions with periods above $\approx 170$ s falling outside the COI. The network regions showed no distinct periods in this line.

The first and brightest network region shows a range of approximately $1.5-5$ cycles, with three short periods having around two cycles and a $77 \mathrm{~s}$ period in $\mathrm{Ne}$ IV with $\approx 4.75 \mathrm{cy}-$ cles; the longer periods tend to cover a range of cycles. Note that hereafter we shall use the term short periods to describe these periods $<100 \mathrm{~s}$.

The inter-network region has between 1.25-4 cycles within the range of detected periods. There are ten short periods present covering a range of cycles from 1.2-3.7. From Fig. 5 
Table 2. Continued.

\begin{tabular}{|c|c|c|c|c|}
\hline Ion Wavelength $\AA$ & Period Range (Max) & Time Range (Max) & Class & Location \\
\hline Ne VIII 770 & $85-291(201)$ & $285 c-1095 c(754)$ & $\mathrm{b}$ & $\mathrm{N} 1$ \\
\hline- & $83-138(100)$ & $1852-2091(1962)$ & $0 \dagger$ & I \\
\hline - & $40-55(47)$ & $2770-2938(2837)$ & o & I \\
\hline - & $56-69(61)$ & $3890-4035$ (3968) & o & I \\
\hline - & $59-85(72)$ & $8066-8267$ (8166) & o & I \\
\hline - & 294-364 (324) & $3386-4203$ (3789) & o & I \\
\hline - & $159-290(236)$ & 9256-9705c (9461) & o & $\mathrm{N} 2$ \\
\hline - & $313-482 c$ & $9126 c-9537 c(9254)$ & $\mathrm{b}$ & $\mathrm{N} 2$ \\
\hline O vi 173 & $36-50(42)$ & $966-1042$ (1000) & o & N1 \\
\hline - & $831-165$ (107) & $519-827$ (627) & $0 \dagger$ & N1 \\
\hline - & $155-470 \mathrm{c}(234)$ & $224-871(366)$ & $\mathrm{b}$ & $\mathrm{N} 1$ \\
\hline - & $125-162(140)$ & $1945-2288(2094)$ & o & I \\
\hline - & $187-283$ (235) & $1670-2176$ (1923) & o & I \\
\hline - & $97-134(118)$ & $8123-8446$ (8303) & o & I \\
\hline - & $173-228(203)$ & $5781-6122(5930)$ & o & I \\
\hline - & $466-969$ (626) & $6445 c-8018 c(7570)$ & $\mathrm{b}$ & I \\
\hline - & $193-277$ (229) & $9010 c-9742 c(9412)$ & $\mathrm{b}$ & $\mathrm{N} 2$ \\
\hline Fe Ix 171 & $185-306(245)$ & $270 c-893(353)$ & $\mathrm{b}$ & $\mathrm{N} 1$ \\
\hline - & 209-247 (228) & $4673-4998(4830)$ & o & I \\
\hline - & $314-542$ (409) & $3184 c-3912(3520)$ & o & I \\
\hline- & $116-482 c(212)$ & $9103 c-9577 \mathrm{c}(9367,9394)$ & $\mathrm{b} \dagger$ & $\mathrm{N} 2$ \\
\hline Fe XI 180 & 79-104 (90) & $6030-6155$ (6092) & o & I \\
\hline - & $99-116(107)$ & $5420-5582(5507)$ & o & I \\
\hline - & $146-215$ (178) & 2929-3465 (3153) & o & I \\
\hline - & $1288-1651(1525)$ & $3950 c-6864 c(5370)$ & $\mathrm{b}$ & I \\
\hline Fe XIII 203 & $75-112(92)$ & $114 c-297(194)$ & o & $\mathrm{N} 1$ \\
\hline - & $205-239(222)$ & $3346-3935$ (3620) & o & I \\
\hline - & $60-155(123)$ & $9135-9619$ (9380) & $0 \dagger$ & I \\
\hline - & $104 c-128(117)$ & $8840 c-9007$ (8864) & o & $\mathrm{N} 2$ \\
\hline- & $228-482 c$ & $9103 c-9705 c(9382)$ & $\mathrm{b}$ & $\mathrm{N} 2$ \\
\hline
\end{tabular}

we find that the majority of the periods are found below $500 \mathrm{~s}$. One possibility is that the periods are limited by the COI; however, due to the greater length of the time series in the internetwork region we would still expect periods up to $\approx 1000 \mathrm{~s}$ to be unaffected by the COI.

The second network region shows a range of 1.5-4.25 cycles. Three short periods are present with 1.5-2.5 cycles with a $72 \mathrm{~s}$ period in Ne vIII having 4.25 cycles. The longer periods tend to lie in the range of 1.5-3.2 cycles. There are a smaller number of distinct periods present in the second network region, appearing to have less cycles than those in the first network region.

Considering Fig. 5 it appears that there are more short periods present in the inter-network region than the network regions. However, the first and second network regions cover the first two and last two positions respectively $(\approx 1300 \mathrm{~s})$, where as the inter-network region covers eleven positions in between
( $\approx 7300 \mathrm{~s}$ ). Thus considering the length of observing time it is possible that the network regions have a greater density of short periods per unit time. It is also possible that some of the short periods fall below the $99 \%$ confidence level in the internetwork, due to the lower intensity level giving a reduced signal to noise ratio.

\subsection{Localised short periods}

The results presented in this paper show a number of short periods $<100$ seconds across the whole temperature range $\log T_{\mathrm{e}}=$ 5.3-6.1 K. The shortest period presented here from Table 2 is $42 \mathrm{~s}$. At a cadence of $\approx 13 \mathrm{~s}$ this would give three data points for one cycle. Here we suggest that for the Morlet wavelet which essentially is a plane wave, that at least five data points are necessary to define an oscillation. The analysis was repeated again with a cadence of $\approx 6$ s to verify that the short periods are real; 
Table 3. Previous work on periodicities within the transition region and corona.

\begin{tabular}{llll}
\hline \hline Author & Region & Spectral range & Period (s) \\
\hline Ireland (1997) & Active Region & EUV & $300,900-1200$ \\
Ireland et al. (1999) & Active Region & EUV & $50-550$ \\
De Moortel et al. (2000) & Active Region & EUV & $180-420$ \\
O'Shea et al. (2001) & Active Region & EUV & $>50,200-500$ \\
Fludra (2001) & Sunspot region & EUV & $120-200$ \\
Doyle et al. (1998) & Quiet Sun & EUV & $200-500$ \\
Hansteen et al. (2001) & Quiet Sun & UV & $>50-140,140-300$ \\
Banerjee et al. (2001a) & Quiet Sun - Network & UV-EUV & $250-500$ \\
Judge et al. (2001) & Quiet Sun - Plage & UV & $140-200$ \\
Banerjee et al. (2001b) & Polar Coronal Hole & EUV & $1200-1800+$ \\
DeForest \& Gurman (1998) & Polar Plume & EUV & $600-900$ \\
McKenzie \& Mullan (1997) & Coronal Loop & X-ray & $10-62$ \\
Aschwanden et al. (1999) & Coronal Loop & EUV & $\approx 280$ \\
Koutchmy et al. (1983) & (Coronagraph) & Green coronal line & $43,80,300$ \\
Rušin \& Minarovjech (1991) & (Coronagraph) & Red/Green coronal line & $40,60,112,300$ \\
Cowsik et al. (1999) & Off-limb (Eclipse) & Optical continuum & $7,25,90$ \\
Singh et al. (1997) & Off-limb (Eclipse) & Optical continuum & $5,6,8,14,20,57$ \\
\hline
\end{tabular}

the $42 \mathrm{~s}$ period then has six data points. All the short periods were still present except for the $59 \mathrm{~s}$ period found in Ne IV. This may be present in the $\approx 13 \mathrm{~s}$ analysis due to the increased signal to noise.

\subsection{Characteristics of significant power}

As mentioned previously wavelet analysis returns frequency information as a function of time. Compared to Fourier methods this gives the advantage that any change in the periods present can be determined. From the wavelet analysis we find a period of $\approx 220$ s present in the first network region in all but the Fe XI and Fe XIII lines. This period may not be present in these lines because two separate time series were formed for the first network region to produce stationary time series. This means that the maximum measurable period would be limited to $\approx 170 \mathrm{~s}$ by the COI. This period appears in the wavelet power spectra as a band of power that stretches across the COI. This band of power also shows a decrease in period with time. Figures 8 and 9 show for each line: The time series (note the different scales on the $y$-axis), wavelet power spectrum with the position of the maximum in the band of power and the change in period of maximum wavelet power with time. Considering Figs. 8 and 9 we can see that the band of power displays a decay in period by $10-20 \%$ in all the lines but one. The exception is O VI which shows a different form than the other lines. $\mathrm{O}$ vi has the lowest signal to noise ratio of all the lines in the study. The difference may be caused by a real structure emitting at this temperature or possibly some subtlety in this line not accounted for in the analysis in Sect. 4.2.1.

If we consider the other lines it appears that the contours of significance represent the same underlying structure in the wavelet power. As explained in Sect. 3.3.1 the contours of significance enclose areas having greater wavelet power than expected for Poisson distributed noise. Thus we would expect the signal to noise ratio to have some effect on the boundary of the contour levels. The Ne vir line has the largest signal to noise ratio of the analysed lines. Examining the wavelet power spectrum for Ne vil in Fig. 8 we see the band of power with the marked maximum and power in a "U" shape below. At the time around $500 \mathrm{~s}$ and at a period of approximately $170 \mathrm{~s}$ we find a small area that drops below the significance level. If we examine the wavelet power spectra for the other lines we find that the wavelet power around this band shows very similar structure. It appears that the underlying wavelet power in this region of the spectrum is almost the same for all the lines but the contours of significance enclose this power to a varying extent. Figures 8 and 9 show that the period causing this band of power is present in these lines at the same point in time and also in location.

\subsubsection{Error analysis}

Figures 8 and 9 show the change of maximum wavelet power with time and the associated error bars. To determine this error it was necessary to measure the error in the ability of the wavelet analysis to determine the correct position of maximum wavelet power. To do this sinusoidal time series were formed to represent the time-dependent $\approx 220 \mathrm{~s}$ band of power for each line. These time series were given a mean equal to the time series mean for each spectral line. The amplitude of the sine wave was then assigned a value to give a variation equal to that of the original data.

Poisson distributed noise was then added to these time series, the wavelet power spectra formed and the position of maximum wavelet power measured. This process was repeated 1000 times to determine the maximum and minimum limits of the position of maximum wavelet power. We would expect the location of maximum wavelet power to be affected by the addition of Poisson noise; the limits then give an estimate of the error in the wavelet analysis method assuming Poisson noise. These limits are then given as the errors on maximum wavelet 

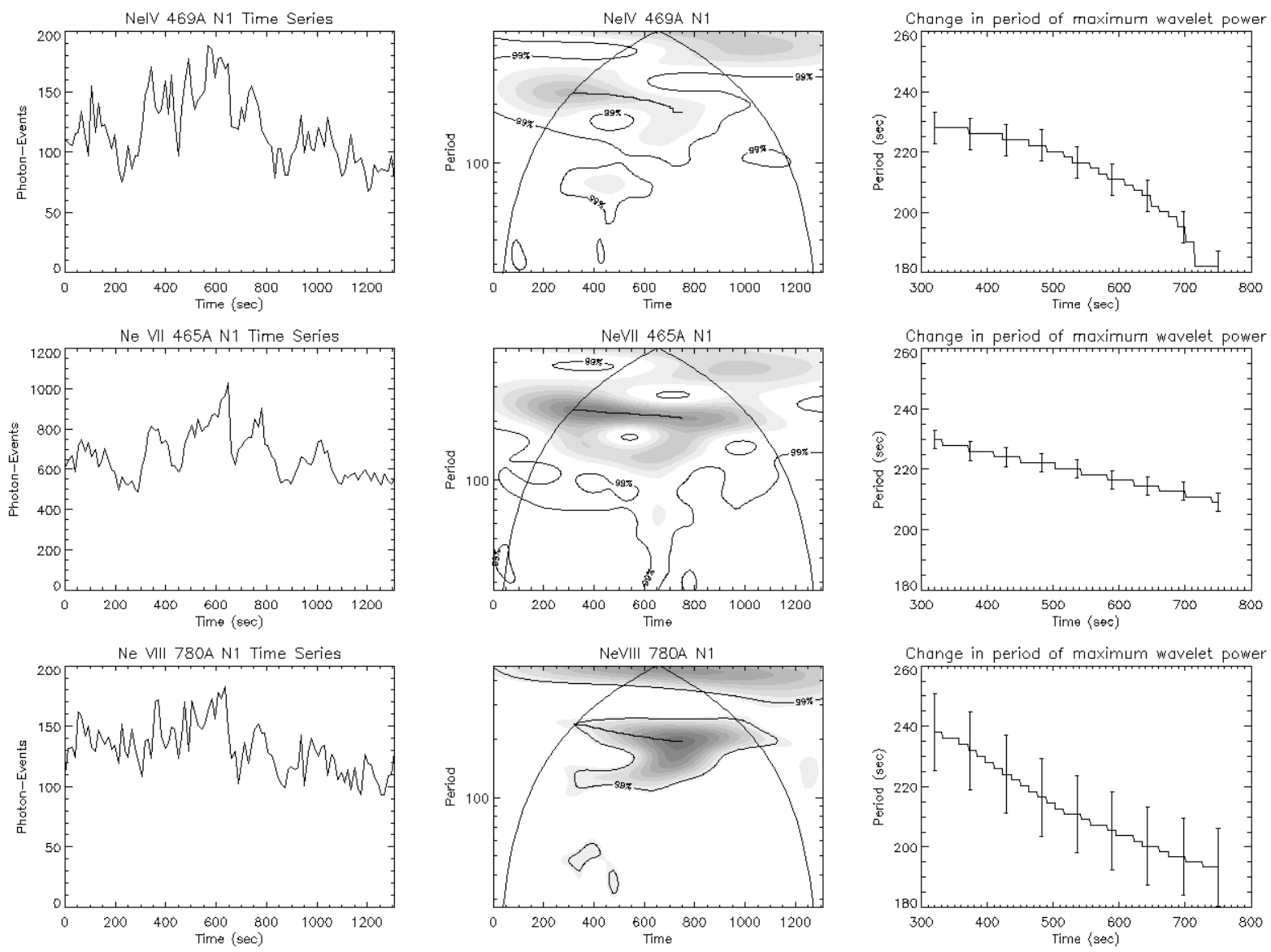

Fig. 8. Characteristics of significant power (N1 region) in Ne IV $469 \AA$, Ne VII $465 \AA$, and Ne VIII $780 \AA$ : time series, wavelet power spectrum and period-time variation of maximum wavelet power.

power for each line, shown in the plots on the right hand side of Figs. 8 and 9.

\section{Discussion}

A wavelet analysis method is employed to investigate oscillations within transition region/coronal spectral lines. The temporal information returned by wavelet analysis allows a deeper investigation of the periodicities present when compared to Fourier methods. The two-dimensional power spectra allows the investigation of oscillations localised in time and the time dependence of any periodicity to be measured. The results presented here reveal periodicities that are consistent with other authors who cover different regions of the sun. Previous work on waves in coronal hole regions (See DeForest \& Gurman 1998; Banerjee et al. 2001b, and references within) concentrate on the observation of polar plumes and report waves with periods of the order of 10-70 min. To our knowledge no previous work has reported the observation of periods within a coronal hole of the order presented here. Original results are also presented of the observation of short wave packets and of a change in driving period with time.

Several authors report on the observation of short periods $<100$ s. Using CDS-NIS (Normal Incidence Spectrometer) active region data Ireland et al. (1999) find short periods of the order $60-70$ s that persist for $2-6$ oscillations in Fe XVI $360 \AA$ $\left(\log T_{\mathrm{e}}=6.4 \mathrm{~K}\right)$ coronal line intensity time series, with no significant short periods in Mg IX $368 \AA\left(\log T_{\mathrm{e}}=6.0 \mathrm{~K}\right)$ or Ov $629 \AA\left(\log T_{\mathrm{e}}=5.3 \mathrm{~K}\right)$. O'Shea et al. (2001) also present results of active region observation using NIS data in the same lines. They find short periods $>50 \mathrm{~s}$ in velocity time series formed from Mg Ix $368 \AA$ and O v $629 \AA$ with no short periods in Fe XVI $335 \AA$. In the intensity time series they find periods $>50 \mathrm{~s}$ in $\mathrm{Mg}$ IX and Fe XvI.

Hansteen et al. (2001) perform intensity and velocity time series analysis using SUMER (Solar UltraViolet Measurements of Emitted Radiation) observations of a quiet sun network/inter-network region using Ca II $1334 \AA$, C III $977 \AA$, and O vi $1032 \AA$ upper chromosphere and transition region lines. Periods between $60-100 \mathrm{~s}$ are found in the intensity signal over a network position; $100 \mathrm{~s}$ periods in intensity are also found in the internetwork region with periods around $60 \mathrm{~s}$ in the velocity signal. They find that short periods are more predominant in $\mathrm{C}$ III and suggest that there is even greater power at short periods in $\mathrm{O}$ vi but the observations are restricted by a high noise level. McKenzie \& Mullan (1997) use Yohkoh X-ray light curves obtained from a number of coronal loops and find periods in the range 10-62 s. Koutchmy et al. (1983), Rušin \& Minarovjech (1991), Cowsik et al. (1999), Singh et al. (1997) all use off-limb observations of the corona either in the red/green coronal lines or optical continuum and find periods in the range 5-90 s. 

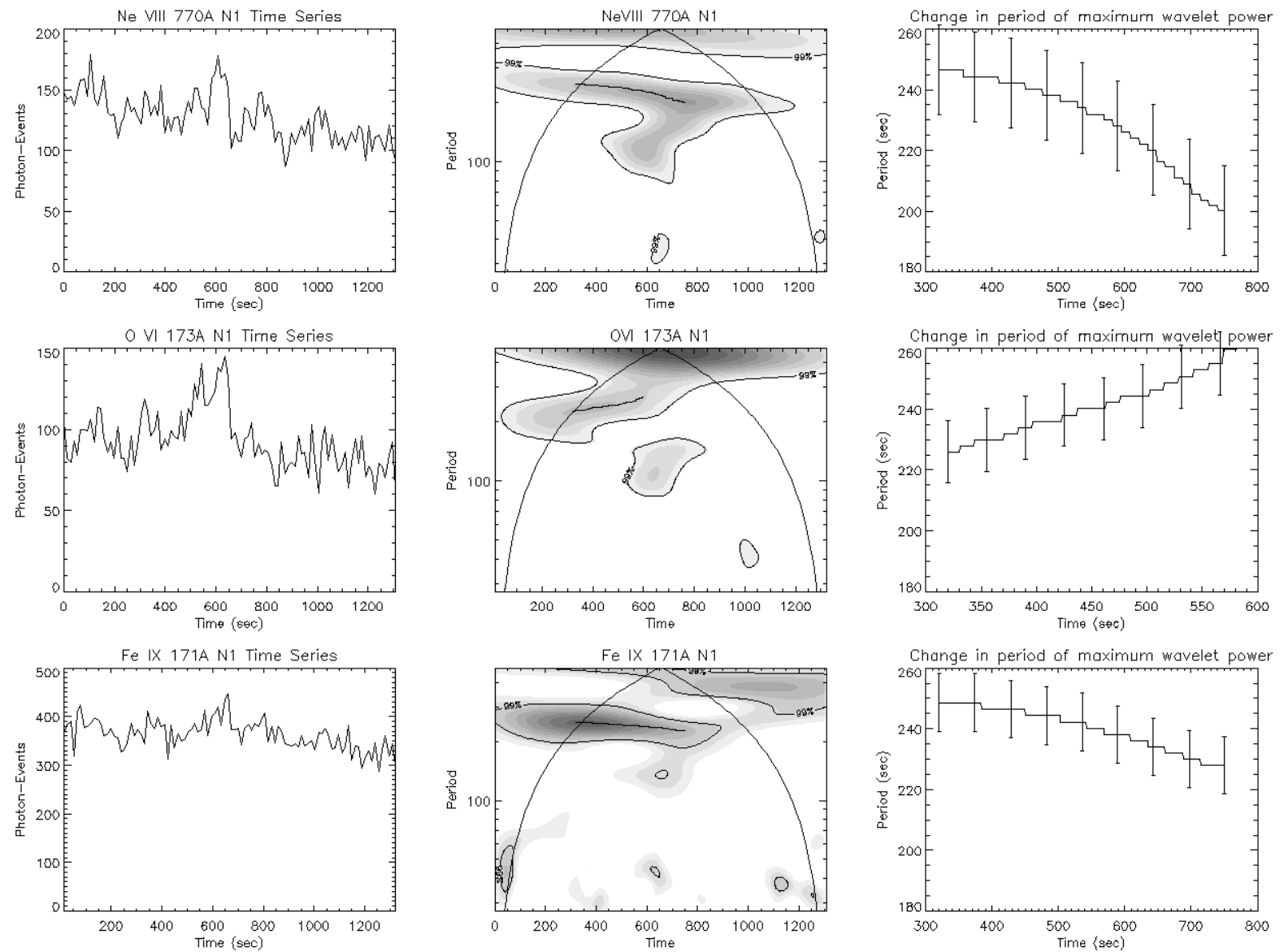

Fig. 9. Characteristics of significant power (N1 region) in Ne vIII $770 \AA$, O vi $173 \AA$, and Fe Ix $171 \AA$ : time series, wavelet power spectrum and period-time variation of maximum wavelet power.

In the previous work listed above short periods are found predominantly in the high temperature coronal lines with the exception of Hansteen et al. (2001) who state that they find power below $100 \mathrm{~s}$ in the upper chromospheric $\mathrm{C}_{\text {II }} 1334$ Å line. It is significant that this current analysis finds short periods in intensity present in lines across the temperature range $\log T_{\mathrm{e}}=$ 5.3-6.1 K. We find short periods that are not confined to coronal temperatures but are also found in the transition region. These short periods are found in both the network and internetwork regions. Considering the length of observing time the short periods appear to show a greater density in time within the network regions. This could be due to a reduced number of drivers operating in the inter-network. Another possibility is that fewer periods are detected due to the lower intensity and therefore lower signal to noise ratio in the inter-network.

We observe a period of $\approx 220 \mathrm{~s}$ in all but the two highest temperature lines over a network region on a coronal hole boundary. This was the only very clear example of a cotemporal oscillation present within different lines. O'Shea et al. (2001) state that they find one of the primary periods present in $\mathrm{Mg}$ IX and Fe XvI at a frequency of $4.5 \mathrm{mHz}(\approx 220 \mathrm{~s})$ in observations of active region oscillations. Hansteen et al. (2001) also find this period in observations of quiet sun regions. We find the magnitude of this period shows a decay with time of $10-20 \%$ throughout the lines. Since the GIS grating is astigmatic and observes one area "on disc" we cannot derive any information about the periodicities as a function of height. It is also difficult to simply use the ion emission temperature to derive height through the transition region and corona. However, we can state that this band of power experiences a decay in the value of its period and the decay of this period is present in a number of lines emitting at temperatures in the range $\log T_{\mathrm{e}}=$ $5.3-6.1 \mathrm{~K}$ at the same point in time and at the same location. This suggests that the driver experiences a decay in its period or there is some change in the physical conditions resulting in the observed time dependence. The periods presented here are consistent with previous work in that they could be interpreted as slow mode magneto-acoustic waves or possibly fast mode in the case of the short periods. Considering the time series in Figs. 8 and 9 no significant change of phase is visible in the $\approx 220$ s period between the different lines. Also, because this band of power is limited by the COI no information on any lag in the start time between different lines can be observed. Another possible cause of this time dependence is the motion of the slit across an inhomogeneous magnetic structure. This implies different locations across the structure would contain waves of slightly different period; this may be a possible signature of phase mixing. This possibility is perhaps less likely considering the smooth decay in period and the fact the data is concatenated from two different positions of the slit to produce the time series for the first network region. Solar rotation can be excluded as a cause of this period as we would expect periods 
on the order of an hour to be caused by material passing under the slit. It is interesting to note that examples of $a \approx 220$ s period can be found in the second network region although these examples are less consistent and extensive in time. It is possible that this may be caused by the lower intensity of the second network region which is located within the coronal hole. Alternatively the observation of this period and its decay in the first network region could be connected to its location on a coronal hole boundary or the higher intensity could possibly indicate some underlying activity.

As is usually the case this work is limited by the resolution of current instruments. For the analysis of short periods the most critical factor is obtaining as high a cadence as possible whilst maximising signal to noise. A higher cadence would increase the data point resolution of the short periods reducing the minimum detectable period and high signal to noise would increase the confidence level available. In this particular work a longer observing time over the network regions would have been useful in observing the time dependent period.

Acknowledgements. M.S. Marsh is supported by a PPARC research studentship. Data provided courtesy of SOHO/CDS consortium. SOHO is a project of international cooperation between ESA and NASA. Wavelet software was provided by C. Torrence and G. Compo, and is available at URL: http://paos.colorado.edu/research/wavelets. Thanks also to J. Ireland for useful discussion and comments on the manuscript.

\section{References}

Aschwanden, M. J., Fletcher, L., Schrijver, C. J., \& Alexander, D. 1999, ApJ, 520, 880

Banerjee, D., O’Shea, E., Doyle, J. G., \& Goossens M. 2001, A\&A, 371,1137

Banerjee, D., O’Shea, E., Doyle, J. G., \& Goossens M. 2001, A\&A, 380, L39
Bentley, R. D. 1999, CDS Software Note No. 54

Breeveld, E. 2000, CDS Software Note No. 55

Breeveld, E. 2000, CDS Software Note No. 56

Cowsik, R., Singh, J., Saxena, A. K., Srinivasan, R., \& Raveendram, A. V. 1999, Sol. Phys., 188, 89

Deforest, C. E., \& Gurman, J. B. 1998, ApJ, 501, L217

Del Zanna, G. 1999, Ph.D. Thesis, University of Central Lancashire, England

De Moortel, I., Ireland, J., \& Walsh, R. W. 2000, A\&A, 355, L23

Doyle, J. G., Van Den Oord, G. H. J., O’Shea, E., \& Banerjee, D. 1998 , Sol. Phys., 181, 51

Fludra, A. 2001, A\&A, 368, 639

Hansteen, V. H., Betta, R., \& Carlsson, M. 2001, A\&A, 360, 742

Harrison, R. A., Sawyer, E. C., Carter, M. K., et al. 1995, Sol. Phys., 163,233

Hollweg, J. V. 1990, Comp. Phys. Rep., 12, 205

Ireland, J. 1995, in Fifth SOHO Workshop: The Corona and Solar Wind Near Minimum Activity, ed. A. Wilson, European Space Agency, p. 433

Ireland, J., Walsh, R. W., Harrison, R. A., \& Preist, E. R. 1999, A\&A, 347,355

Judge, P. G., Tarbell, T. D., \& Wilhelm, K. 2001, AJ, 554, 424

Koutchmy, S., Žugžda, Y. D., \& Locăns, V. 1983, A\&A, 120, 185

McKenzie, D. E., \& Mullan, D. J. 1997, Sol. Phys., 176, 127

Nakariakov, V. M., \& Ofman, L. 2001, A\&A, 372, L53

Narain, U., \& Ulmschneider, P. 1990, Space. Sci. Rev., 54, 377

O'shea, E., Banerjee, D., Doyle, J. G., Fleck, B., \& Murtagh, F. 2001, A\&A, 368, 1095

Robbrecht, E., Verwichte, E., Berghmans, D., et al. 2001, A\&A, 370, 591

Rušin, V., \& Minarovjech, M. 1991, in Mechanisms of Chromospheric and Coronal Heating, ed. P. Ulmschneider, E. R. Preist, \& R. Rosner (Springer-Verlag, Berlin), p. 30

Singh, J., Jagdev, C. R., \& Raveendran, A. V., et al. 1997, Sol. Phys., 170,235

Torrence, C., \& Compo, G. P. 1998, Bull. Amer. Meteor. Soc., 79, 61

Zirker, J. B. 1993, Sol. Phys., 148, 43 